\title{
La nostalgie des origines dans Le Procès-verbal de J.M.G. Le Clézio
}

\author{
Denis Bachand \\ Université d'Ottawa
}

Écrire, c'est surtout essayer de survivre. Le Clézio, Télérama (13 décembre 2000)

Il m'arrivera souvent d'apprécier un tableau uniquement par la somme d'idées ou de rêveries qu'il apportera dans mon esprit.

Baudelaire (Écrits sur l'art, p. 380)

Roman fondateur de l'œuvre de Le Clézio, Le Procès-verbal (1963) préfigure l'ensemble des réseaux thématiques et symboliques d'une écriture en quête de ses propres origines. Roman de la régression, roman initiatique, roman-puzzle d'une 
trajectoire de retour et d'enroulement de la conscience dans la spirale de la contemplation, Le Procès-verbal est tout cela à la fois et davantage encore : une expérience d'écriture excavatrice au parcours mythique qui tente de donner sens à l'aventure de vivre. Appuyée sur la poétique bachelardienne, la symbolique alchimique et la psychologie jungienne, cette étude vise à exhumer l'écheveau intertextuel crypté sous-jacent au récit.

\section{La vie refusée : le repli}

«Voilà comment j'avais rêvé de vivre depuis des temps. » (PV, p. 14) Ainsi débute la première lettre d'Adam à Michèle. Par cette confidence épistolaire s'inscrit le schéma archétypal de «la maison onirique » au cœur du processus de retour à la matrice originelle (Bachelard, p. 95). L'abri rêvé est une retraite, une protection contre le bruit et la dispersion. L'être rêvant y trouve le repos de l'âme propice à l'expansion dynamique de l'imaginaire : " [...] il s'exerçait à imaginer [...] », précise l'instance narrative hétérodiégétique dès le premier chapitre $(P V$, p. 20). Un premier chapitre inaugural à un double titre puisqu'il s'ouvre sur la première lettre de l'alphabet: la lettre A. Celle qui pose dès le départ la relation au langage comme une énigme à résoudre et un ordre à suivre. Un ordre au double sens de l'institution d'une autorité combinatoire dans et par laquelle s'inscrit le sujet du discours et d'un ordonnancement de la succession linéaire du dire en action. Tous les autres chapitres seront eux aussi coiffés de ces repères alphabétiques élémentaires qui segmentent le récit, évitent la dispersion et intriguent tout à la fois puisqu'on ne sait trop s'il s'agit d'un message crypté ou si ce n'est qu'un simple jeu destiné à confondre le lecteur en faisant mine de le guider. Il 
reste que l'énigme est posée et balisée par les signes minimaux du matériau de base de l'expression qui institue le verbe en procès, qui le met en demeure de raconter en substituant ses signes aux données perceptibles du monde phénoménal. En d'autres mots, le code linguistique s'institue en médiateur de la représentation en intimant son ordre et en imposant son système de classement; c'est lui qui mène le jeu.

La maison abandonnée est un repère, à la fois lieu protecteur et dispositif qui offre des indices sur la voix/voie à suivre. Adam, le premier des hommes selon la tradition biblique, créé à partir de la prima materia que l'alchimiste Dorneus appelle «Adamica» (pareille à Adam) (Jung, 1970, p. 408), s'y réfugie et s'y love pour ainsi dire métaphoriquement en position de retour aux sources pour y accueillir l'indicible qui le conduira "vers les mystérieuses obscurités de son langage naissant » ( $P V$, p. 248), vers le b.a.-b.a. embryonnaire de l'énonciation qui le constituera en tant que sujet d'une quête identitaire, dans le chaos de la matière brute du langage pour y accomplir son œuvre au noir. Car, si «Le Clézio rêve de plénitude et souhaite se fondre dans le monde[, l]a création littéraire devient alors le moyen privilégié de pallier cette déficience cosmique » (Favre, p. 167). En quittant la ville sur sa moto, Adam abandonne l'espace de la multiplicité, de l'éclatement et de l'égarement pour chercher l'apaisement, le calme et le repos dans la solitude d'une maison vide au bord de l'élément fécondateur premier, la mer. L'isolement se pose ainsi comme condition première du retour aux sources, du retour à soi. À l'altérité menaçante de la multitude, du dehors, se substituent progressivement les profondeurs abyssales de l'intériorité où Adam s'abîme en contemplation, s'imaginant luimême en imaginant la matière, dans les plis et replis de laquelle 
se projettent la profondeur de son mystère et celle de tout vivant. Ce travail d'introspection révèle un spectacle fantastique et hallucinant où grouillent en abondance des créatures de toutes sortes dans un univers dominé par un "soleil noir », véritable inversion symbolique qui témoigne de l'accomplissement de la régression au cœur de la matière philosophale. Comme l'a montré Gilbert Durand, les symboles de l'intimité sont profondément marqués par «l'isomorphisme du retour, de la mort et de la demeure » (p. 69). Il n'y aura plus d'obscurité quand l'imagination aura vaincu l'opacité des apparences, quand le regard intérieur aura vaincu les obstacles des sens, quand l'anima aura rejoint l'animus l'Adam hermaphrodite des alchimistes), quand le yin et le yang se fusionneront dans l'abolition des contraires pour réaliser l'unité perdue des origines : "C'est en réconciliant le Père et la Mère, le conscient masculin et l'inconscient féminin, que la psyché engendre le fils philosophique.» (Mariel, p.110) Le désir d'immersion se traduit ainsi par une écriture faisant appel aux figures de résorption des paradoxes, ce qui est le propre du mythe qui se pose en métadiscours de médiation et de résorption des contradictions. La rêverie n'a plus qu'à s'inscrire dans la voie de l'hyperperception où, pour reprendre la pensée de Jung à propos de la fonction transcendante, Adam connaît les «épousailles dans l'être de son conscient et de son inconscient » (1964, p. 207) :

$\mathrm{Au}$ plus profond d'Adam, c'était l'agglomérat de cellules, de noyaux, de plasma, d'atomes aux combinaisons multiples; plus rien n'était étanche. Les atomes d'Adam auraient pu se mêler aux atomes de la pierre, et lui, s'engloutir très doucement à travers terre et sable, eau et limon; tout aurait croulé ensemble, comme dans un gouffre, et se serait évanoui parmi le noir. $(P V$, p. 230-231) 


\section{La vie contemplée : l'imagination de la matière}

Pour retourner la matière, il faut remonter le temps. En laissant s'échapper l'autre monde, Adam arrive ainsi «à recomposer un univers de terreurs enfantines» $(P V$, p. 21$)$ : «Le petit Adam a bientôt douze ans; le soir, dans la ferme, pendant qu'il pleut dehors, [...] il prend un grand carton bleu et il dessine le monde. » (PV, p. 201-202) Comme l'affirme Bachelard, « [s]ur le chemin qui nous mène aux origines, il y a d'abord le chemin qui nous rend à notre enfance, à notre enfance rêveuse qui voulait des images, qui voulait des symboles pour doubler la réalité. » (p. 122) C'est aussi ce que fait le jeune Adam qui, « [e]n haut du carton bleu, à gauche, [fait] une boule rouge et jaune avec ses crayons de couleur; c'est comme le soleil, à ceci près qu'il n'y a pas de rayons. [...] Puis il fait une ligne droite qui barre le carton au-dessous du soleil-lune et de la lune-soleil.» ( $P V$, p. 202) Deux figures paradoxales, un chiasme qui résorbe les contraires dans la fusion symbolique des antagonismes, processus qui est le propre du récit mythique. L'imagination travaille ainsi la matière du langage pour éliminer les oppositions et retrouver l'unité (la coincidentia oppositorum des alchimistes) indistincte à la base du développement originaire du fœetus humain dans l'ordre biologique et qui trouve écho dans l'ordre psycho-symbolique: «C'est en ce sens que l'inconscient, dans ses formes les plus primitives, est hermaphrodite», nous rappelle Bachelard (p. 149). Dans un pareil cheminement intérieur où imagination et sensations altèrent les perceptions, la chronologie disparaît dans les échappées de la mémoire affective. Comme pour la madeleine de Proust, le passage du temps s'abolit dans les espaces retrouvés et réintégrés des jeux d'enfance alors que la dynamique créatrice du langage tente de donner formes à la mémoire des sens : 
Ça faisait qu'il se sentait en équilibre, sur la pointe des pieds, perché tout en haut des continents, tenant sous ses semelles une terre ronde comme une mappemonde, imitant la posture de Marie et inversant le travail d'Atlas; semblable à l'époque (douze-treize ans) où de toute la force de son poids, il obligeait la sphère de caoutchouc à demeurer plongée sous la mer, dilatée par la pression, et remontant vaguement par petites glissades le long de ses mollets. ( $P V$, p. 44-45)

La situation ainsi évoquée de l'inversion du travail d'Atlas et de la posture de Marie peut se représenter par la figure du sceau de Salomon (figure du Grand CEuvre réalisé) formée de deux triangles inversés symbolisant le féminin et le masculin, le feu et l'eau, synonymes également de la sagesse humaine dans sa quête d'unité (voir Chevalier et Gheerbrant, p. 769). Le retour sur le chemin de l'enfance et de la naissance est aussi une quête d'un sens transcendant qui passe paradoxalement par la contemplation de l'inhérent, au cœur de la matière. Dans son refuge, entre deux chaises longues vides, Adam observe le monde par une fenêtre ouverte, et ce sont des images du fond de sa mémoire qui surgissent: "à force de voir le monde, le monde lui était complètement sorti des yeux » $(P V$, p. 89). Mais l'isolement et la solitude sont difficiles à assumer: "Ça demande de savoir s'organiser, [...] d'avoir envie de creuser des tanières, tout le temps, et de s'y fourrer, tout humilié, bien au secret, comme on faisait quand on était gosse, entre deux pans crevés de vieille bâche. » $(P V$, p. 25) Lové au creux rassurant et protecteur d'un lieu secret, tous les ingrédients d'un retour symbolique à la cavité utérine sont réunis pour illustrer combien la retraite dans la maison abandonnée sert d'incubation à la parole. La maison devient ainsi l'équivalent du vase merveilleux (vas mirabile), qui « est une sorte de matrice ou d'utérus d'où doit naître le filius philosophorum (fils des 
philosophes), la pierre miraculeuse. » (Jung, 1970, p. 309-310) C'est en ce sens que l'on doit comprendre, tel un Zarathoustra, son discours devant la populace: "Vous vendez quelque chose?" dit une femme. "Oui, la parole", dit Adam. » (PV, p. 247) De la même façon qu'il est impossible de sortir physiquement par ses yeux pour naître au-dehors, il est impossible pour Adam de s'accoucher extérieurement par la parole; il réintègre alors son cocon : «il retourna vers les mystérieuses obscurités de son langage naissant, vers son isolement forcené [...]. » (PV, p. 248) De cette façon, la parole aussi suit un processus involutif pour disparaître dans la région du pré/dit, du pré-natal : « Il pensait peut-être, tout bas, tout ténu, longtemps avant ses cordes vocales gelées, qu'il était bien dans son domaine. » ( $P V$, p. 312) L'aphasie guette du dedans alors que l'imagination construit des êtres monstrueux qui indiquent bien qu'il « savait qu'il était dans la bonne voie » ( $P V$, p. 21), dans celle de l'animalisation que Bachelard associe à l'accentuation de la descente (ou de l'ascension vers les origines) dans les rêveries du repos :

[...], et ils vinrent en rangs serrés, à travers la campagne, cannibales, lâches ou féroces. Les papillons de nuit s'acharnèrent sur son corps, le mordirent de leurs mandibules, l'enveloppèrent du voile soyeux de leurs ailes velues. Des mares surgit tout un peuple caparaçonné, de parasites ou de crevettes, de crustacés brusques, mystérieux, avides de lui arracher des morceaux de chair. Les plages furent couvertes d'êtres bizarres, qui venaient $y$ attendre on ne sait quoi [...]. (PV, p. 21)

\section{La vie révélée : le corps-texte}

Dans l'ordre symbolique, l'animalisation des fantasmes correspond à une progression dans l'âme du rêveur sur le chemin qui conduit à la « maison-corps » (Bachelard, p. 126) où 
fusionnent indistinctement le dehors et le dedans, bien avant que le stade du miroir lacanien n'ait accompli son œuvre d'objectivation et d'autonomisation du sujet. Aussi les figures du bestiaire ne sont-elles qu'une étape menant à la vision de l'unicité et de la fluidité de la matière microscopique qui traverse et donne forme à l'ensemble de la création. La faculté imageante du langage, sa poésie (sa poïèsis) enroule l'être dans le compost du monde où sont indistinctement réunis et confondus les règnes animal, végétal et minéral; il va de même pour Adam pour qui «la conscience de la vie n'est que la connaissance nerveuse de la matière» $(P V$, p. 28). Ne coule bientôt plus qu'une seule énergie :

Après cela, ce furent les trous dans les rochers; il y en avait de toutes les tailles, des grands et des moins grands; ils en choisirent un de taille normale, un trou à une place, et s'y vautrèrent de tout leur long. Surtout Adam : il ne passait pas de jour sans accomplir cette merveille : excitant au paroxysme son sens mythologique, il s'entourait de pierres, de décombres; il aurait aimé avoir tous les détritus et ordures du monde pour s'y ensevelir [...] il se dissimulait dans les fissures du sol, et attendait, béatifié, que quelque eau le germe. ( $P V$, p. 75)

Après la maison, l'animal et la matière, c'est le propre corps d'Adam qui est objet de méditation : «Au plus profond d'Adam, c'était l'agglomération de cellules, de noyaux, de plasma, d'atomes aux combinaisons multiples; plus rien n'était étanche.» ( $P V, \quad$ p. 230) Dans cette suite d'exercices de visualisation, il y a nettement accentuation de la progression vers l'intimité, vers l'intimité universelle : «Les atomes d'Adam auraient pu se mêler aux atomes de la pierre, et lui, s'engloutir très doucement à travers terre et sable, eau et limon [...]. » $(P V$, p. 230). Ainsi, la matrice s'agrandit aux dimensions de l'univers, Adam s'imagine (ou s'hallucine?) fœtus cosmique avant de 
régresser totalement dans la pré-vie ou la mort: " Il est dans l'huître, et l'huître au fond de la mer. » ( $P V$, p. 314) Avec la disparition des frontières corporelles, c'est toute l'assurance identitaire du moi individuel (de l'ego) qui s'abolit dans l'indistinction fondamentale de la matière cosmique; c'est aussi la voie mystique, celle d'une parole qui s'évertue à se taire pour rejoindre son horizon de silence. On peut dire qu'Adam est parvenu à la «fermeture des premiers repos» (Bachelard, p. 149) dans le ventre sphérique de la conscience parménidienne qui lui est si chère $(P V, \mathrm{p} .68-69)$. En réclusion volontaire dans une chambre qui a toutes les caractéristiques d'un ventre protecteur et nourricier, bien à l'abri de la figure solaire paternelle, Adam (le premier des hommes, duquel, selon le mythe, naquit la première des femmes) s'apprête à donner naissance à une Ève intérieure: une tentation de fusion hermaphrodite dans l'espace du silence. Une quête ultime de sens, une quête d'absolu qui transfigure le réel au point de créer de véritables visions anamorphiques qui s'imposent au regard halluciné du rêveur (du drogué ou du malade?) pour qui les carreaux de la fenêtre de la chambre prennent la forme de la Huitième Maison du Ciel ( $P V$, p. 258), celle de la mort selon le poète-astronome latin Manilius ( $1{ }^{\mathrm{er}}$ siècle), celui à qui l'on doit la formule selon laquelle « [e]n naissant, nous mourons, et la fin dépend de l'origine ». Derrière les lèvres et les cordes vocales gelées où «il n'avait plus grand-chose à écrire » $(P V, 265)$, Adam l'aphasique s'imagine " [rétrogradant] dans le sang et le pus, jusqu'au ventre de sa mère, où, bras et jambes en posture de l'œuf, l'on s'endort la tête contre la membrane de caoutchouc, d'un sommeil obscur peuplé d'étranges cauchemars terrestres » $(P V$, p. 314) : «Ce qu'on vit, ce qu'on écrit, c'est contre sa mère », écrira plus tard Le Clézio dans 
L'Extase matérielle (p. 189); contre et « tout contre », pourrionsnous ajouter.

\section{La vie originelle : une mystique de la parole}

L'image du repliement fœtal rejoint celle de la métaphore de l'œuf philosophique, «lieu et sujet de toutes les transmutations» (Chevalier et Gheerbrant, p. 554) : «En alchimie, l'œuf représente le chaos tel que le conçoit l'adepte, la prima materia dans laquelle l'âme du monde est captive » (Jung, 1970, p. 266). Bachelard emprunte cette voie interprétative dans sa réflexion sur les rêveries du repos: "Ainsi l'imagination minutieuse veut se glisser partout, elle nous invite non seulement à rentrer dans notre coquille, mais à nous glisser dans toute coquille pour y vivre la vraie retraite, la vie enroulée, la vie repliée sur soi-même, toutes les valeurs du repos. » (Bachelard, p. 18) Ainsi, le projet de se retrouver à l'état de germe prêt à être fécondé à nouveau peut se traduire par une quête de renaissance au sein du creuset de la matière première, dans sa froide et pourtant rassurante immobilité minérale et cristalline: "Je vivrais bien au milieu d'une montagne de cailloux blancs et d'une jungle incendiée [...]. Comme un bloc de glace du Pôle Nord, ça serait l'harmonie matérielle, grâce à quoi le temps ne coule plus. » ( $P V$, p. 211). L'œuvre au noir réalisée permet l'accomplissement de l'œuvre au blanc et de l'œuvre au rouge. Toujours ces images de fixité et de pérennité que le langage érige pour endiguer la fluidité, la dispersion et l'éphémère de toutes choses. Car pour Le Clézio, celui de L'Extase matérielle, " [l]'écriture est la seule forme parfaite du temps» (p. 104). Une formule mystérieuse, énigmatique et lapidaire qui fait écho aux exercices de visualisation ou de 
méditation inspirées des pratiques orientales, de la drogue et des voyages astraux qui procurent les expériences extatiques de la découverte de la simultanéité que recherche si vivement Adam et que les alchimistes représentaient par la figure circulaire de l'ouroboros, le dragon qui se mord la queue en signe de négation du temps (voir Jung, 1970, p. 377). Il s'établit ainsi une équivalence entre ces deux ordres de pratiques, celle de l'écriture et celle de la méditation sous toutes ses formes qui cherche à répondre à la question existentielle fondamentale du sens de la vie et de la mort. Une écriture qui se cherche et se forge par l'exploration et l'emprunt aux textes de différentes traditions scientifiques, symboliques et ésotériques pour traduire le spectacle de la réalité (de l'Être vrai de Parménide), de ce qui se révèle dans ces instants de fulgurance méditative (ou d'extase matérialiste) que dévoile le maelström de la matière en devenir; d'où ces efforts de précision du langage dans un désir irréaliste de tout retenir « [puisque l'on] ne finira jamais de raconter la création de la terre » (La Guerre, p. 288).

Le mot, là, se tait, impuissant à cerner et à nommer, à contenir l'expérience de la transparence existentielle inter/dite dans la fracture de la parole et de la pensée. Le mot aussi doit se dissoudre pour rejoindre l'autre côté de la langue telle une radioscopie révélatrice de l'ordre du dedans des choses et des corps. C'est pourquoi «Le Clézio a constamment recours au paradoxe, frustré par l'effort vain de décrire l'ineffable, de communiquer l'inédit» (Holzberg, p. 155). Le territoire à nommer est atteint par une surcharge effrénée de paroles, domaine de la littérature en lutte contre et pour le rétablissement de l'ordre du réel. Sans les signes, le temps ne coule plus; on peut vivre au milieu du blanc fixe et éternel. L'œuf représente cette image où cohabitent l'Alpha et l'Oméga, 
« l'être d'être » $(P V$, p. 300) où l'on est « définitivement brûlant [rouge], définitivement créé » ( $P V$, p. 212, raturé dans le texte); et aussi définitivement non-créé (ou incréé), d'où cette rature dans le texte qui signifie bien que l'œuf représente le lieu du positif et du négatif, de la vie et de la mort conjointement, la coincidentia. Le lieu de la transparence est celui où plus rien ne s'écrit, où l'écrit se rature, s'efface au même instant qu'il s'inscrit en vaine tentative d'endiguer le cours du temps qui glisse... entre les mots. Car, ainsi que le formulera plus tard Le Clézio dans L'Extase matérielle, «[c]omme la mort est le parachèvement de la vie, ce qui lui donne forme et valeur, ce qui ferme sa boucle, de même le silence est l'aboutissement suprême du langage et de la conscience. Tout ce que l'on dit ou écrit, tout ce que l'on sait, c'est pour cela, pour cela vraiment : le silence.» (p. 92) Ne demeure alors plus qu'une volonté de disparition dans le grand tout cosmique. Yves-Alain Favre le souligne : "Pourtant, le salut existe : il faut parvenir à l'“extase matérielle" qui consiste à abolir les frontières entre le moi et l'univers [...]. Face à un univers hostile et menaçant, dont l'homme n'a su qu'aggraver et amplifier les conflits latents. Le Clézio réagit par un désir de fusion avec les éléments naturels. » (p.168 et 180) Ce qui explique la quête d'Adam, quête mue à la fois par une pulsion de mort et un espoir de renaissance: "J'espère qu'on ne reconnaîtra plus rien de moi dans cette momie gercée. Parce que je voudrais bien vivre tout nu et tout noir $[\ldots] »(P V$, p. 67). La dispersion dans la matière (CEuvre au Noir) participe ainsi d'une volonté alchimique de renaissance où la terre devient un fourneau (un athanor) où les éléments chauffés (à rouge et à blanc) peuvent féconder le nouvel Adam: "Il se centrait au milieu de la matière, de la cendre, des cailloux, et peu à peu se statufiait. » $(P V$, p. 75$)$ 
Celui qui « [aurait] préféré ne jamais être né » (La Fièvre, p. 7), celui qui « [n'est] pas vraiment sûr d'être né » (La Guerre, p. 289), celui qui voudrait « habiter dans des ventres de femme, pour être bien » (Les Géants, p 109), celui qui écrit « qu'il faut chercher loin, à l'envers, [...] fouiller le passé. Retrouver le père qu'on n'a pas connu, la mère qui ne nous a pas enfanté » (Le Livre des fuites, p. 9), celui-là s'emploiera dans la suite de son œuvre à remonter le temps par l'intermédiaire d'une imagination nourrie aux récits des générations passées pour se donner une identité grâce à l'écriture. C'est en remontant le cours du temps et des générations que Le Clézio s'adonnera à une véritable archéologie de la mémoire qui le conduira à creuser, non seulement ses propres souvenirs, mais aussi ceux de ses ancêtres, de ceux et celles qui l'ont précédé. Désert (1980), Le Chercheur d'or (1985), Onitsha (1991), Étoile errante (1992), Révolutions (2003), L'Africain (2004), Ritournelle de la faim (2008) entre autres, sont imprégnés de ces récits multiples et entrelacés qui donnent naissance et consistance à son écriture; autant d'exhumations de la mémoire ancestrale grâce à laquelle se construit progressivement, et comme en un seul texte, le récit identitaire de l'écrivain qui poursuit inlassablement sa mise au monde. 


\section{Bibliographie}

BACHELARD, Gaston. (1963), La Terre et les rêveries du repos, Paris, José Corti.

BAUDELAIRE, Charles. (1971), Écrits sur l'art, t. I, Paris, Gallimard, coll. « Folio » 3135.

CHEVALIER, Jean et Alain GHEERBRANT. (1969), Dictionnaire des symboles, Paris, Robert Laffont.

DURAND, Gilbert. (1969), Les Structures anthropologiques de l'imaginaire, Paris, Bordas, coll. «Études supérieures ».

FAVRE, Yves-Alain. (1989), « Le Clézio. L'expérience du cosmos et l'écriture », $S U D$, no 85/86, p. 167-180.

JUNG, Carl Gustav. (1964), Dialectique du moi et de l'inconscient, Paris, Gallimard, coll. « Idées » 285;

- (1970), Psychologie et alchimie, Paris, Buchet/Chastel.

HOLZBERG, Ruth. (1981), L'œil du serpent. Dialectique du silence dans l'œuvre de J.-M.G. Le Clézio, Sherbrooke, Naaman.

Le Clézio, J.M.G. ([1963]1970), Le Procès-verbal, Paris, Gallimard, coll. « Folio » 2748;

—. (1965). La Fièvre, Paris, Gallimard, coll. « Le chemin »;

—. ([1967] 1971). L'Extase matérielle, Paris, Gallimard, coll. «Folio » 239;

—. (1970). La Guerre, Paris, Gallimard, coll. « Le chemin »;

—. (1973). Les Géants, Paris, Gallimard, coll. « Le chemin »;

-. (2004). L'Africain, Paris, Mercure de France, coll. « Traits et portraits ».

MARIEL, Pierre. (1970), « Jung et l'alchimie », Planète Plus, no 18 , p. 105-111. 


\section{Résumé}

Prix Nobel de littérature 2008, le prolifique Jean-Marie Gustave Le Clézio est considéré comme un écrivain majeur dès la parution de son premier roman Le Procès-verbal, pour lequel on lui décernera le Prix Renaudot en 1963. Tout à la fois roman de la régression et de la quête initiatique, Le Procès-verbal préfigure les thématiques qui seront développées par la suite dans l'ensemble de son œuvre. Le présent article en propose une lecture psycho-mythique fondée sur la poétique bachelardienne, la symbolique alchimique et la psychologie jungienne.

\section{Abstract}

Prolific author and 2008 Nobel Prize winner in literature, JeanMarie Gustave Le Clézio was immediately recognized as a major writer with his first novel Le Procès-verbal, for which he received the Prix Renaudot in 1963. In many regards, Le Procèsverbal became a preview of - and reflects - the themes explored and developed in the whole of his future writings. Using Bachelard's poetic, alchemist symbolism and Jungian psychology as a foundation for interpretation, this article offers a psycho-mythological reading of Le Clézio's first important contribution to literature. 